\title{
Analysis of cytotoxic $T$ lymphocytes from a patient with hepatocellular carcinoma who showed a clinical response to vaccination with a glypican-3-derived peptide
}

\author{
YOSHITAKA TADA $^{1,2}$, TOSHIAKI YOSHIKAWA ${ }^{1}$, MANAMI SHIMOMURA ${ }^{1}$, YU SAWADA ${ }^{1}$, MAYUKO SAKAI ${ }^{1}$, \\ HIROFUMI SHIRAKAWA $^{1}$, DAISUKE NOBUOKA ${ }^{1}$ and TETSUYA NAKATSURA ${ }^{1,2}$ \\ ${ }^{1}$ Division of Cancer Immunotherapy, Research Center for Innovative Oncology, National Cancer Center Hospital East, \\ Kashiwa, Chiba 277-8577; ${ }^{2}$ Research Institute for Biomedical Sciences, Tokyo University of Science, \\ Noda, Chiba 278-0022, Japan
}

Received June 3, 2013; Accepted July 12, 2013

DOI: 10.3892/ijo.2013.2044

\begin{abstract}
Glypican-3 (GPC3), which is a carcinoembryonic antigen, is overexpressed in human hepatocellular carcinoma (HCC). Previously, we performed a phase I clinical trial of GPC3-derived peptide vaccination in patients with advanced HCC, and reported that GPC3 peptide vaccination is safe and has clinical efficacy. Moreover, we proposed that a peptide-specific CTL response is a predictive marker of overall survival in patients with HCC who receive peptide vaccination. In this study, we established GPC3-derived peptide-specific CTL clones from the PBMCs of an HLA-A*02:07-positive patient with HCC who was vaccinated with an HLA-A2-restricted GPC3 peptide vaccine and showed a clinical response in the phase I clinical trial. Established CTL clones were analyzed using the IFN- $\gamma$ ELISPOT assay and a cytotoxicity assay. GPC3 peptide-specific CTL clones were established successfully from the PBMCs of the patient. One CTL clone showed cytotoxicity against cancer cell lines that expressed endogenously the GPC3 peptide. The results suggest that CTLs have high avidity, and that natural antigen-specific killing activity against tumor cells can be induced in a patient with HCC who shows a clinical response to vaccination with the GPC $3_{144-152}$ peptide.
\end{abstract}

\section{Introduction}

Primary liver cancer, which is frequently hepatocellular carcinoma (HCC), is the sixth most common cancer and third most frequent cause of cancer-related death worldwide, and it is becoming more prevalent not only in East Asia, South-East Asia, and Africa but also in Western countries (1-3). Recently,

Correspondence to: Dr Tetsuya Nakatsura, Division of Cancer Immunotherapy, Research Center for Innovative Oncology, National Cancer Center Hospital East, 6-5-1 Kashiwanoha, Kashiwa, Chiba 277-8577, Japan

E-mail: tnakatsu@east.ncc.go.jp

Key words: glypican-3, peptide vaccine, CTL clone the multikinase inhibitor sorafenib was demonstrated to prolong overall survival (OS) in patients with advanced HCC, and it has become the standard drug for first-line systemic treatment (4-6). However, based on the Response Evaluation Criteria in Solid Tumors (RECIST), the response rate for sorafenib is rather low, and the incidence of adverse events is relatively high, especially in elderly patients (7). Therefore, the generation of a novel effective therapy for HCC is a priority.

Immunotherapy is an attractive option for treating HCC. Many of the tumor antigens associated with HCC are potential candidates for peptide vaccines $(8,9)$. The carcinoembryonic antigen Glypican-3 (GPC3), which is a $65-\mathrm{kDa}$ protein of 580 amino acids, belongs to the family of glycosyl-phosphatidylinositol (GPI)-anchored heparan sulfate proteoglycans (HSPG) $(10,11)$. GPC 3 is specifically overexpressed in HCC (72-81\% of cases) and correlates with poor prognosis (12-16). This suggests that GPC3 is an ideal target for anti-HCC immunotherapy.

We have previously demonstrated the antigenicity of GPC3, and that the HLA-A*24:02-restricted GPC $3_{298-306}$ (EYILSLEEL) peptide and the HLA-A*02:01-restricted GPC $_{144-152}$ (FVGEFFTDV) peptide can induce GPC3-reactive CTLs without inducing autoimmunity (17-21).

HLA-A2 is the most frequent HLA-A type in all ethnic groups (22). HLA-A2 is also expressed in about $40 \%$ of Japanese persons $(23,24)$ and in about $50 \%$ of Caucasians (25). Among Caucasians, $>90 \%$ of HLA-A2-positive individuals carry the HLA-A*02:01 allele (25), whereas among the Japanese, there are multiple common and well-documented (CWD) allelic variants, including HLA-A*02:01, HLA-A*02:06 and HLA-A*02:07 (26). The frequencies of the HLA-A*02:01, HLA-A*02:06 and HLA-A*02:07 alleles in the Japanese population are 19, 14 and 7\%, respectively (26). Therefore, we confirmed that the HLA-A*02:01-restricted GPC $3_{144-152}$ (FVGEFFTDV) peptide could also bind to HLA-A*02:06 and HLA-A*02:07 using a binding assay (unpublished data).

On the basis of these results, we conducted a phase I clinical trial of a GPC3-derived peptide vaccine in 33 patients with advanced HCC. The HLA-A*24:02-restricted GPC $3_{298-306}$ peptide was used for HLA-A*24:02-positive patients and 
the HLA-A*02:01-restricted GPC $3_{144-152}$ peptide was used for HLA-A*02:01, HLA-A*02:06 and HLA-A*02:07-positive patients. We found that GPC3 vaccination was well-tolerated, and that the GPC3 peptide vaccine induced a GPC3-specific CTL response in almost all of the patients (27-30). Moreover, the vaccination-induced GPC3-specific CTL response correlated with overall survival (OS); the OS was significantly longer in patients with high GPC3-specific CTL frequencies than in those with low GPC3-specific CTL frequencies (27). In terms of clinical responses, one patient showed a partial response (PR) and 19 patients showed stable disease 2 months after initiation of treatment. One patient with $\mathrm{HCC}$ who showed a PR was HLA-A*02:07-positive. In addition, several HLA-A*02:01-restricted GPC3 peptide-specific CTL clones with cytotoxic activities against GPC3 were established from the peripheral blood mononuclear cells (PBMCs) of patients vaccinated in this trial (27).

The aims of the present study were: i) to establish GPC3-derived, peptide-specific CTL clones from the PBMCs of an HLA-A*02:07-positive patient with HCC who showed a PR in the phase I clinical trial; and ii) to analyze the functions of these CTL clones.

\section{Materials and methods}

Ethics information. This study was approved by the Ethics Committee of the National Cancer Center and conformed to the ethical guidelines of the 1975 Declaration of Helsinki. All the patients gave written informed consent before entering the study at the National Cancer Center Hospital East (Chiba, Japan). The trial has been registered with the University Hospital Medical Information Network Clinical Trials Registry (UMIN-CTR no. 000001395).

PBMCs collection. Peripheral blood samples were obtained pre- and post-vaccination from the patient with HCC who was HLA-A*02:07-positive. Post-vaccination, blood samples were collected from the patient every 2 weeks. The GMP-grade peptide GPC $3_{144-152}$ (FVGEFFTDV) (American Peptide Co., Sunnyvale, CA, USA) was emulsified in IFA (Montanide ISA-51 VG; SEPPIC, Paris, France) and injected intradermally at $30 \mathrm{mg} /$ body three times at 14-day intervals $(27,28)$. PBMCs were isolated by density centrifugation using Ficoll-Hypaque (Pharmacia, Uppsala, Sweden) and frozen in liquid nitrogen until use.

Cell lines. The human lung cancer cell line 1-87 (GPC3-, HLA-A ${ }^{*} 02: 07^{+} / \mathrm{A}^{*} 11: 01^{+}$) and hepatitis B virus (HBV)-integrated human hepatocellular carcinoma cell line JHH-7 (GPC ${ }^{+}$, HLA-A*24:02 $2^{+} / \mathrm{A}^{*} 31: 01^{+}$) were conserved in our laboratory and cultured in Dulbecco's modified Eagle's medium (DMEM; Sigma Chemical Company, St. Louis, MO, USA) that was supplemented with $10 \%$ heat-inactivated fetal bovine serum (FBS; Gibco, Carlsbad, CA, USA).

Plasmids and transfection. The expression vectors pcDNA3.1 (Invitrogen, Carlsbad, CA, USA) and pcDNA3.1 that contained the HLA-A*02:07 cDNA were used for the transfection experiments. The pcDNA3.1 construct that contained HLA-A*02:07 was kindly provided by Dr Ryo Abe
(Tokyo University of Science, Chiba, Japan). The JHH-7/ HLA-A*02:07 cell line was obtained by transfection of JHH-7 cells with the expression vector using FuGENE HD (Roche Applied Science, Mannheim, Germany). JHH-7/mock and JHH-7/HLA-A*02:07 cells were cultured in DMEM that was supplemented with $10 \%$ heat-inactivated FBS and $1 \mathrm{mg} / \mathrm{ml}$ G418 (Calbiochem, Darmstadt, Germany).

Induction of GPC $3_{144-152}$ peptide-specific CTLs from PBMCs. The PBMCs were cultured $\left(2 \times 10^{6}\right.$ cells/well) with the GPC $3_{144-152}$ peptide in RPMI-1640 (Sigma Chemical Company) that was supplemented with $10 \%$ heat-inactivated FBS, $100 \mathrm{IU} / \mathrm{ml}$ recombinant human IL-2 (Nipro, Osaka, Japan), and $10 \mathrm{ng} / \mathrm{ml}$ recombinant human IL-15 (PeproTech Inc, Rocky Hill, NJ, USA) for 14 days.

CD107a staining and flow cytometry analysis. $\mathrm{CD}^{+} \mathrm{T}$ cells were isolated using human CD8 microbeads (Miltenyi Biotec, Bergisch Gladbach, Germany) from PBMCs that were stimulated with the GPC $3_{144-152}$ peptide for 14 days. The $\mathrm{CD}^{+} \mathrm{T}$ cells were incubated with GPC $3_{144-152}$-pulsed or $\mathrm{HIV}_{19-27}$-pulsed 1-87 cells at a ratio of $2: 1$ for $3.5 \mathrm{~h}$ at $37^{\circ} \mathrm{C}$. CD107a-specific antibodies (BD Biosciences, San Jose, CA, USA) were included in the mixture during the incubation period.

Generation of CTL clones. $\mathrm{CD}^{+} \mathrm{CD} 107 \mathrm{a}^{+}$cells were sorted using a FACSAria cell sorter (BD Biosciences). Sorted CTLs were stimulated and the CTL clones were established as previously described (28).

Cytotoxicity assay. Cytotoxic capacity was analyzed with the Terascan VPC system (Minerva Tech, Tokyo, Japan). The CTL clone was used as the effector cell type. Target cells were labeled in calcein-AM solution for $30 \mathrm{~min}$ at $37^{\circ} \mathrm{C}$. The labeled cells were then co-cultured with the effector cells for 4-6 h. Fluorescence intensity was measured before and after the culture period, and specific cytotoxic activity was calculated as previously described (28).

IFN- $\gamma$ ELISPOT assay. Specific secretion of IFN- $\gamma$ from human CTLs in response to stimulator cells was assayed using the IFN- $\gamma$ ELISPOT kit (BD Biosciences), according to the manufacturer's instructions. Stimulator cells were pulsed with or without peptide for $1.5 \mathrm{~h}$ at room temperature and then washed three times. Responder cells were incubated with stimulator cells for $20 \mathrm{~h}$. The resulting spots were counted using an ELIPHOTO counter (Minerva Tech).

Determination of recognition efficiency. Calcein-AM-labeled target cells were pulsed with various concentrations of peptide, starting at $10^{-6} \mathrm{M}$ and decreasing in log steps to $10^{-14} \mathrm{M}$. The CTL clones were incubated with the target cells at an effector:target $(\mathrm{E} / \mathrm{T})$ ratio of $10: 1$ for $4 \mathrm{~h}$. The recognition efficiencies of the CTL clones were defined as previously described (28).

RNA interference. Human GPC3-specific siRNAs were chemically synthesized as double-strand RNA (Invitrogen). A non-silencing siRNA, AllStras Neg. Control siRNA, was obtained from Qiagen (Valencia, CA, USA). The following 
GPC3-specific siRNA sequences were used: GPC3-siRNA (\#4149), 5'-UUAUCAUUCCAUCACCAGAGCCUCC-3'; GPC3-siRNA (\#4150), 5'-GGAGGCUCUGGUGAUGGAAU GAUAA-3': and GPC3-siRNA (\#4151), 5'-UAUAGAUGACUG GAAACAGGCUGUC-3'. Synthetic siRNA duplexes were transfected using Lipofectamine RNAiMAX (Invitrogen), according to the manufacturer's protocols.

$R T-P C R$. Using the TRIzol reagent (Invitrogen), we extracted total cellular RNA from untreated or siRNA (GPC3-siRNA or negative-siRNA)-treated JHH-7/HLA-A*02:07. cDNA was synthesized using the PrimeScript II 1st Strand cDNA Synthesis kit (Takara, Kyoto, Japan) according to the manufacturer's instructions. The cDNA was added to a reaction mix that contained 10X Ex Taq Buffer (Takara), $2.5 \mathrm{mM}$ dNTP mixture (Takara), 5 units Ex Taq (Takara), and $10 \mu \mathrm{M}$ of the GPC3- or $\beta$-actin-specific PCR primers. The following primer sequences (sense and antisense, respectively) were used: for GPC3, 5'-AGCCAAAAGGCAGCAAGGAA-3' and 5'-AAGA AGAAGCACACCACCGA-3'; and for $\beta$-actin, 5'-CCTCGCCT TTGCCGATCC-3' and 5'-GGATCTTCATGAGGTAGTC AGTC-3'. PCR was performed using the 96-well Gene Amp PCR System 9700 (Applied Biosystems, Carlsbad, CA, USA). PCR was performed for 20 cycles of $98^{\circ} \mathrm{C}$ for $10 \mathrm{sec}, 64^{\circ} \mathrm{C}$ for $30 \mathrm{sec}$ and $72^{\circ} \mathrm{C}$ for $30 \mathrm{sec}$, followed by a step of $72^{\circ} \mathrm{C}$ for $10 \mathrm{sec}$.

Sequence analysis of TCR- $\beta$ gene. Using the TRIzol reagent (Invitrogen), total cellular RNA was extracted from established CTL clones. The cDNA of the TCR- $\beta$ gene was synthesized using the PrimeScript II 1st Strand cDNA Synthesis Kit (Takara) according to the manufacturer's instructions, with the modification that we used $200 \mathrm{nM}$ of the primer specific for the TCR- $\beta$ chain constant region. The cDNA products were subjected to 2 -step PCR, as previously described by Yukie Tanaka-Harada $(35,36)$, and the PCR products were purified and sequenced in the Applied Biosystems 3500 Genetic Analyzer (Applied Biosystems). The $T C R-\beta$ variable $(T R B V)$ gene, $T C R-\beta$ joining (TRBJ) gene, $T C R-\beta$ diversity $(T R B D)$ alleles, and complementarity-determining region 3 $(C D R 3)$ sequences were identified using the IMGT databases (http://www.imgt.org/).

\section{Results}

GPC $3_{144-152}$ peptide-specific CTLs in the peripheral blood of the patient exert a clinical effect. We analyzed the immune responses of the patient who showed a PR following GPC $3_{144-152}$ peptide vaccination. In this patient, the supraclavicular lymph node metastases markedly regressed, two liver tumors disappeared, and the thoracic bone metastasis showed necrosis after the third vaccination (27). The levels of DCP decreased in the patients over the 2-month period. We evaluated the GPC $3_{144-152}$-specific immune responses in the peripheral blood using the ex vivo IFN- $\gamma$ ELISPOT assay. For the HLA-A*02:07-positive patient with advanced HCC, the number and area of the spots increased after two rounds of vaccination, as compared with the pre-vaccination values, and the peak values were noted 10 weeks after the start of the treatment (Fig. 1A).

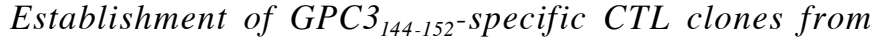
the PBMCs of the patient. To investigate the ability of the GPC $3_{144-152}$-specific CTLs induced by peptide vaccination to recognize antigen, we established CTL clones from the PBMCs of this patient 10 weeks after the start of treatment. The PBMCs were stimulated with the GPC $3_{144-152}$ peptide in vitro for 14 days. $\mathrm{CD}^{+} \mathrm{T}$ cells were isolated from the stimulated PBMCs, and then incubated with peptide-pulsed $1-87$ cells. $\mathrm{CD}^{+} \mathrm{CD} 107 \mathrm{a}^{+}$ cells that reacted with the GPC $3_{144-152}$-pulsed 1-87 cells were sorted to the single-cell level. Thus, we established GPC $3_{144-152}$ peptide-specific CTL clones.

Three established CTL clones were analyzed for function using the IFN- $\gamma$ ELISPOT assay and cytotoxicity assay. All of the CTL clones released IFN- $\gamma$ in response to the GPC $3_{144-152}$-pulsed 1-87 cells, but not in response to non-pulsed 1-87 cells (Fig. 1B). Moreover, these CTL clones showed cytotoxicity against GPC $3_{144-152}$-pulsed 1-87 cells, but not against non-pulsed or HIV19-27-pulsed 1-87 cells (Fig. 1C). These results indicate that the CTL clones 24-4-2, 24-4-7 and 24-2-10 have specificity for the GPC $3_{144-152}$ peptide.

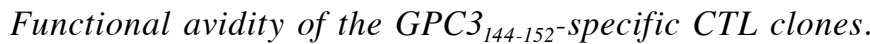
We evaluated the cytotoxicity profiles of the CTL clones for 1-87 cells pulsed with a decreasing concentration series (from $10^{-6}$ to $10^{-14} \mathrm{M}$ ) of the GPC $3_{144-152}$ peptide. The peptide concentration at which the curve reached $50 \%$ cytotoxicity was defined as the recognition efficiency of the clone. The recognition efficiencies of CTL clones 24-4-2, 24-4-7 and 24-2-10 were $10^{-11}, 10^{-9}$ and $10^{-8} \mathrm{M}$, respectively (Fig. 2). This result suggests that CTL clone 24-4-2 has a higher avidity than the other two clones and, conversely, that CTL clone 24-2-10 has a lower avidity than the other two clones.

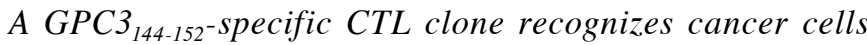
that endogenously express GPC3. Next, we tested the reactivities of these CTL clones against cancer cell lines that expressed GPC3 and HLA-A*02:07. We used the JHH-7/mock (GPC3 ${ }^{+}$, HLA-A*02:07-) and JHH-7/HLA-A*02:07 (GPC3 ${ }^{+}$, HLA-A ${ }^{*} 02: 07^{+}$) transfectants as the target cells (Fig. 3A). The CTL clone 24-4-2 (with high avidity) produced IFN- $\gamma$ and was cytotoxic for JHH-7/HLA-A*02:07 cells but not for JHH-7/mock cells (Fig. 3B and C). The other clones did not produce IFN- $\gamma$ and did not exhibit cytotoxicity for the two target cell lines. These results suggest that only high-avidity CTLs recognize cancer cells that express GPC3 peptide endogenously.

CTL clone 24-4-2 shows specificity for GPC3. To ascertain the GPC3 antigen-specific response of CTL clone 24-4-2, we created a GPC3 knockdown via siRNA treatment of the JHH-7/HLA-A*02:07 cells. GPC3 expression by the JHH-7/HLA-A*02:07 cells was clearly decreased by the GPC3-siRNA, as assessed by RT-PCR (Fig. 4A). We examined the IFN- $\gamma$ production levels of CTL clone 24-4-2 against JHH-7/HLA-A*02:07 cells treated with GPC3-siRNA. IFN- $\gamma$ production by CTL clone 24-4-2 was significantly decreased by the GPC3-siRNA (Fig. 4B). These results indicate that the HLA-A2-restricted GPC $3_{144-152}$ peptide is processed naturally by cancer cells, and that both HLA-A*02:07 and HLA-A*02:01 can present the GPC $3_{144-152}$ peptide on the surfaces of cancer cells. 


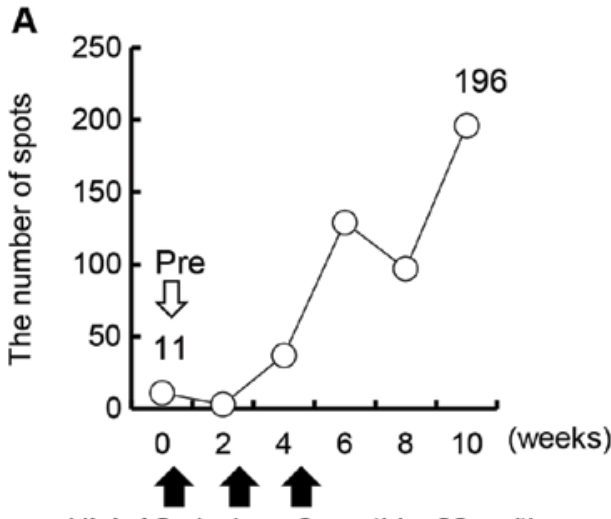

HLA-A2 glypican-3 peptide: $30 \mathrm{mg} /$ time

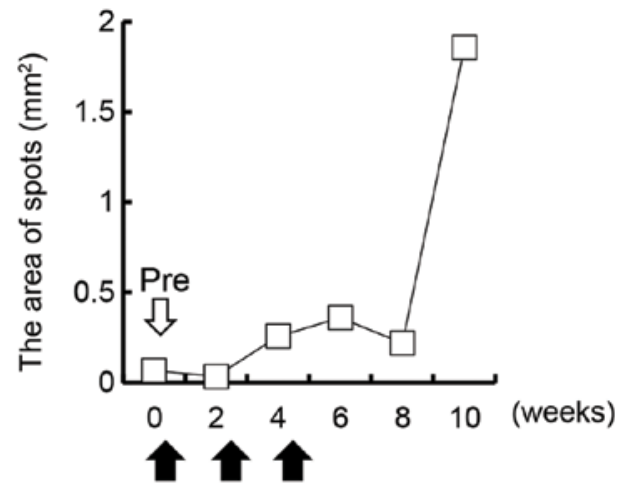

HLA-A2 glypican-3 peptide: $30 \mathrm{mg} /$ time
B

$$
\text { clone 24-4-2 }
$$

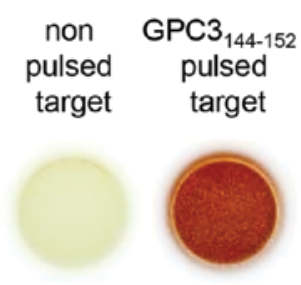

C

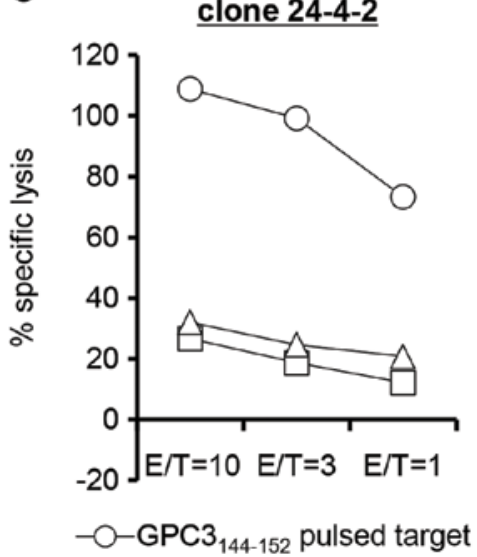

clone 24-4-7
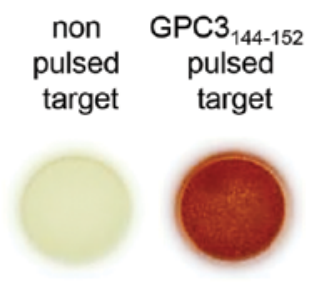

clone 24-4-7

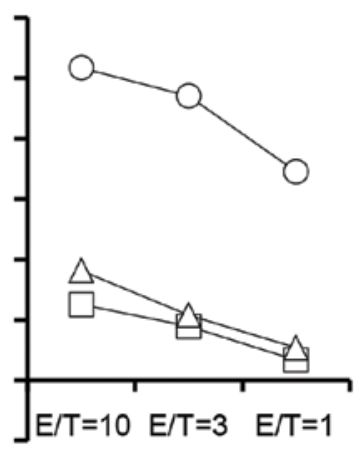

- HIV $19-27$ pulsed target clone 24-2-10

$\begin{array}{rc}\text { non } & \text { GPC3 }_{144-152} \\ \text { pulsed } & \text { pulsed } \\ \text { target } & \text { target }\end{array}$
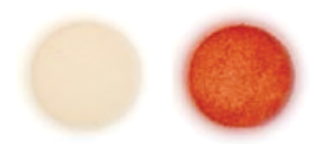

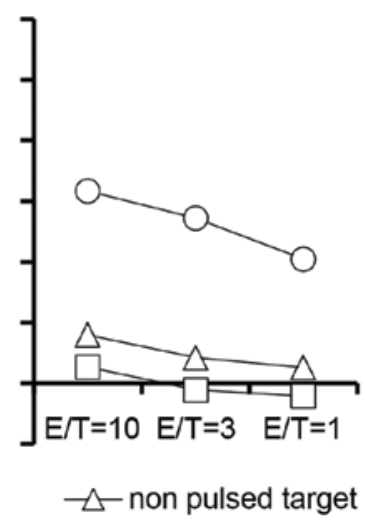

Figure 1. GPC3 peptide-specific CTL clones established from the PBMCs of a patient following GPC3 peptide vaccination. (A) Changes in the frequencies of GPC $_{144-152}$ peptide-specific CTLs before and after vaccination in a patient who showed a PR post-vaccination. Changes in the GPC 3 peptide-specific CTLs are observed as differences in the number (left) and the area (right) of spots in an ex vivo IFN- $\gamma$ ELISPOT assay. (B) Results of the IFN- $\gamma$ ELISPOT assay against peptide-pulsed target. HLA-A*02:07+ cancer cell line 1-87 was used as the target. The target was pulsed with the GPC $3_{144-152}$ peptide. A non-pulsed target was used as the negative control. The ratio of effector cells to target cells (E/T) is 1. (C) Results of the cytotoxicity assay against peptide-pulsed target. The 1-87 cells were used as the target. Non-pulsed and $\mathrm{HIV}_{19-27}$ peptide-pulsed targets were used as negative controls. E/Ts are 10, 3 and 1, respectively. A representative of three experiments is shown.

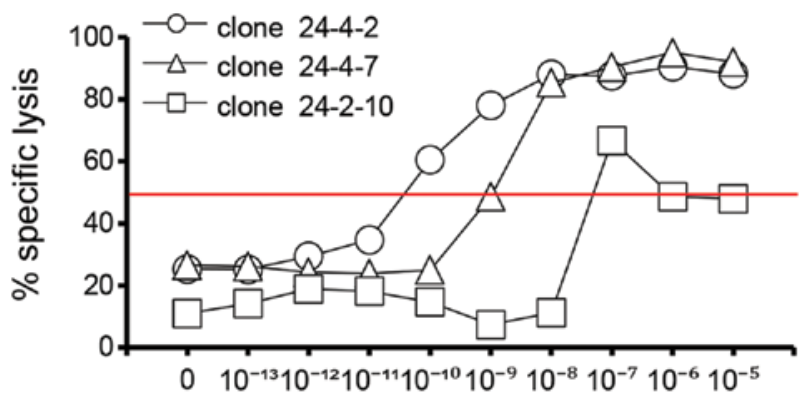

Peptide concentration (M)
Figure 2. GPC $3_{144-152}$ peptide-specific avidity of the established CTL clones. The established CTL clones were tested for avidity using 1-87 cells that were pulsed with various concentrations of the GPC $3_{144-152}$ peptide. The peptide concentration at which the curve crossed the $50 \%$ cytotoxicity mark was defined as the recognition efficiency of that clone. $\mathrm{E} / \mathrm{T}$ is 10 . A representative of three experiments is shown.

Established CTL clones have different sets of TCR- $\beta$ alleles. We analyzed the TCR- $\beta$ gene sequences of the established CTL clones. The TRBV, TRBJ and TRBD alleles were identified using the IMGT databases. Thus, we identified the $T R B V$, $T R B D$ and TRBJ alleles of the CTL clones (Table I). Each of the established CTL clones had different allele sets. 
A
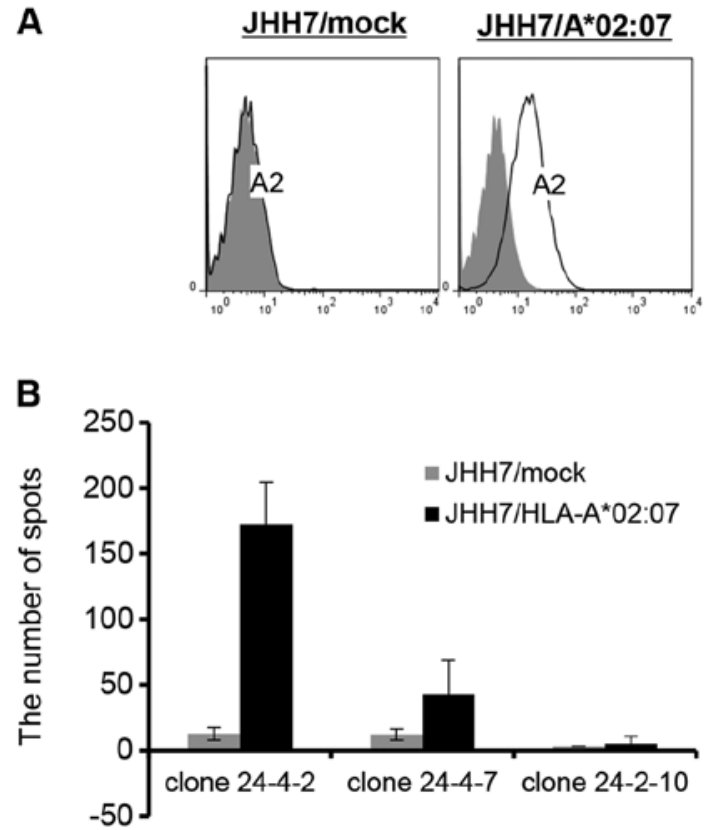

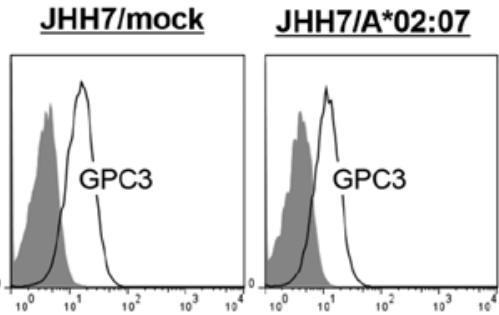

C

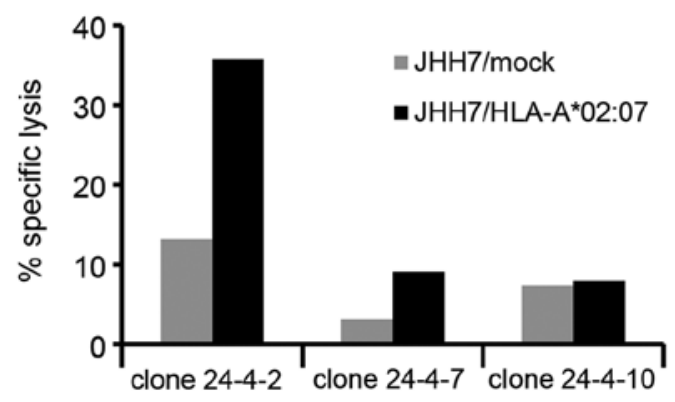

Figure 3. Recognition of GPC $3^{+}$cancer cells by the established CTL clones. (A) Expression of HLA-A2 (left panel) and GPC3 (right panel) on established GPC $3^{+}$ HLA-A ${ }^{*} 02: 07^{+}$cancer cells and control cells. (B) Results of the IFN- $\gamma$ ELISPOT assay for the GPC $3^{+}$cancer cell line. The HLA-A* $02: 07-0 v e r e x p r e s s i n g ~ G P C 3^{+}$ cancer cell line, JHH7/HLA-A*02:07, was established and used as the target. JHH7/mock cells were used as the negative control. E/T ratio, 1 . Data are presented as mean \pm SD of three independent batches. (C) Results of the assay for cytotoxicity against the GPC $3^{+}$cancer cell line. JHH7/HLA-A*02:07 cells were used as the target. JHH7/mock cells were used as the negative control. E/T is 3 . A representative of three experiments is shown.

A

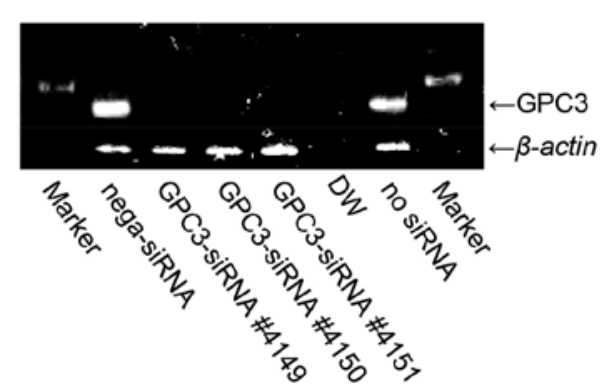

B

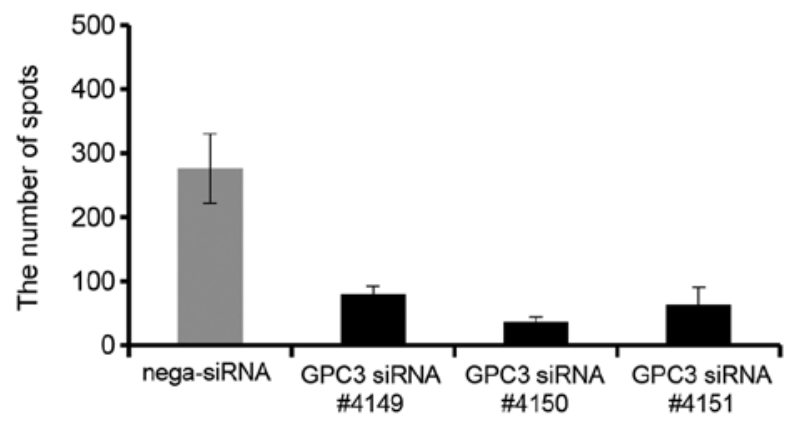

Figure 4. GPC3 specificity of CTL clone 24-4-2. (A) GPC3 expression levels on JHH7/HLA-A*02:07 cells treated with GPC3-siRNA or negative (nega)-siRNA for $48 \mathrm{~h}$, as determined by RT-PCR. (B) Results of the IFN- $\gamma$ ELISPOT assay for JHH7/HLA-A*02:07 cells treated with GPC3-siRNA or nega-siRNA. E/T is 1. Data are presented as mean \pm SD of three independent batches.

CTL clone 24-4-2 is subject to HLA-A*02:07 restriction. We investigated whether CTL clone 24-4-2 recognized the GPC $3_{144-152}$ peptide-HLA-A*02:01 complex and the GPC $3_{144-152}$ peptide-HLA-A*02:06 complex, as well as the GPC $3_{144-152}$ peptide-HLA-A*02:07 complex. Healthy donor PBMCs with HLA-A*02:01, HLA-A*02:06, HLA-A*02:07 and HLA-A*24:02 were used as the targets, and an HLA-A*02:01-restricted, GPC3-specific CTL clone, which is a previously established CTL clone (26), was used as the control. The HLA-A*02:01-restricted CTL clone recognized only the GPC $3_{144-152}$ peptide-HLA-A*02:01 complex, and CTL clone 24-4-2 recognized only the GPC $3_{144-152}$ peptide-HLA-A*02:07 complex (Fig. 5). These outcomes indicate that CTL clone 24-4-2 has HLA-A*02:07 restriction.
Table I. TCR- $\beta$ chain sequencing for established CTL clones.

\begin{tabular}{lccc}
\hline No. & TRBV & TRBJ & TRBD \\
\hline Clone 24-4-2 & $18^{*} 01$ & $1-2^{*} 01$ & $1^{*} 01$ \\
Clone 24-4-7 & $7-3^{*} 01$ & $2-7^{*} 01$ & $1^{*} 01$ \\
Clone 24-2-10 & $7-6^{*} 01$ & $2-1^{*} 01$ & $2^{*} 01$ \\
\hline
\end{tabular}

\section{Discussion}

Clinical trials of peptide-based vaccines are underway in several parts of the world. However, the monitoring of individual CTL post-vaccination has scarcely been reported in 


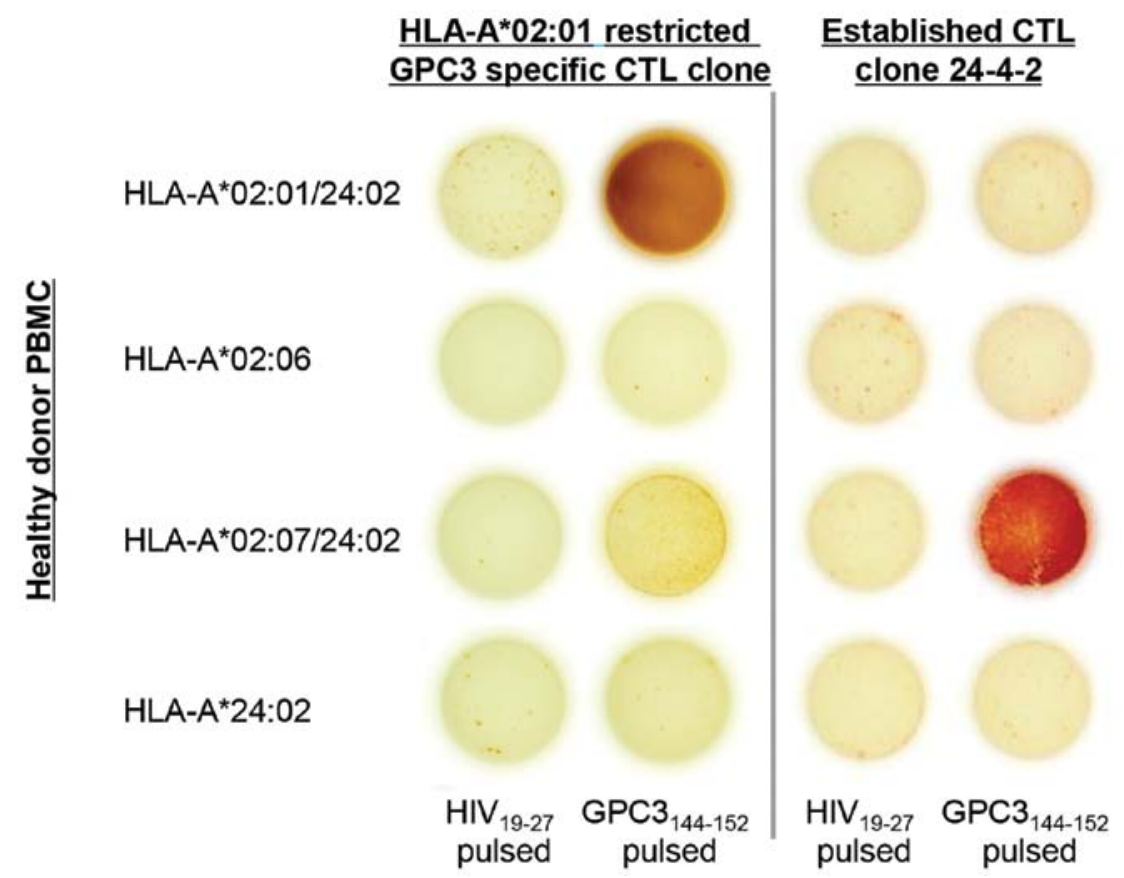

Figure 5. CTL clone 24-4-2 shows HLA-A*02:07 restriction. Results of the IFN- $\gamma$ ELISPOT assay for healthy donor PBMCs with HLA-A2. The established CTL clone 24-4-2 and the HLA-A*02:01-restricted, GPC3-specific CTL clone were used as effectors. E/T is 0.2. A representative of two experiments is shown.

immunotherapy trials. In the present study, we established HLA-A*02:07+ GPC $_{144-152}$-specific CTL clones from the PBMCs of a patient who showed a PR following GPC3-derived peptide vaccination and we performed functional analyses against established CTL clones.

This patient showed an increase in the number of CTLs specific for the GPC3-derived peptide in the peripheral blood after vaccination (Fig. 1A) (27,28). Ten weeks after the start of treatment, the GPC $3_{144-152}$-specific CTL counts had increased approximately 18 -fold, as compared with the pre-vaccination counts. In this case, analysis of the established CTL clones after vaccination could lend support to the notion that the vaccine-induced CTLs exert an antitumor effect, since few GPC $3_{144-152}$-specific CTLs were detected before vaccination.

In the present study, we confirmed that GPC $3_{144-152}$-specific CTL clones are cytotoxic for both GPC $3_{144-152}$-pulsed 1-87 cells and JHH-7/HLA-A*02:07 cells that express GPC3 peptide endogenously. Confirming that the GPC3 peptide-specific CTL clones kill cancer cells that express endogenously the antigen peptide is important because antigen-derived and CTL-inducible peptides are not necessarily presented by cancer cells that endogenously express the antigen (31-33). Three established CTL clones showed cytotoxic activities related to their avidity for GPC $3_{144-152}$-pulsed 1-87 cells and JHH-7/ HLA-A*02:07 cells that expressed the GPC3 peptide endogenously. These results show that although CTLs with different avidity can be isolated, only those CTLs with high avidity can kill cancer cells that express the antigen peptide endogenously. Several investigators have demonstrated a correlation between T-cell avidity and target recognition by $\mathrm{T}$-cell populations that recognize murine tumor models and human cancers (34). Our results strongly support this observation.

The TCR usage of antigen-specific T cells is thought to be influenced by the affinity of the TCR for the antigen
peptide-HLA class I complex. Several studies on the TCR usage of tumor-associated antigen (TAA)-specific T cells have used the $T R B V$ gene family (35-41). These studies mainly analyzed the frequencies of TAA tetramer positive $\mathrm{CD}^{+}$ $\mathrm{T}$ cells. Although it is important to examine quantitative aspects, such as the frequencies of TAA tetramer positive $\mathrm{CD}^{+}$ T cells, the cytotoxicity of these T cells against cancer cells that express the TAA peptide endogenously cannot be confirmed. Moreover, GPC3 dextramer positive $\mathrm{CD}^{+} \mathrm{T}$ cells were not detected in the PBMCs of the patients with HCC before GPC3 peptide vaccination $(27,28)$. To analyze biased usage of the $T C R$ gene of GPC3 dextramer positive $\mathrm{CD}^{+} \mathrm{T}$ cells in the patients with $\mathrm{HCC}$ before and after GPC3 peptide vaccination, a new detection system with greater sensitivity ex vivo will be required. In the present study, we analyzed the $T C R-\beta$ genes of the established GPC $3_{144-152}$-specific CTL clones, to confirm that these CTL clones have different TCRs. Our experiments show that the established CTL clones have different TCR- $\beta$-chain allele sets, i.e., TRBV, TRBD and TRBJ alleles (Table I), and different CDR3 sequences (data not shown). These results suggest that various GPC3-specific CTLs are induced by GPC $3_{144-152}$ peptide vaccination.

A*HLA-A*02:07 differs from HLA-A*02:01 by a single non-conservative change ( $\mathrm{Y}$ to $\mathrm{C}$ ) at residue 99 . X-ray crystallographic data have identified position 99 as one of the residues forming the $\mathrm{D}$ secondary pocket, which engages the residue at position 3 on peptide ligands (42-44). Although hHLA-A*02:07 was originally not included in the HLA-A2 supertype, cross-reactivity between HLA-A*02:07 and other A2 subtypes was detected at the functional level $(44,45)$. Moreover, this HLA molecule indeed binds a subset of the peptide repertoire bound by other A2 subtypes (44). For these reasons, HLA-A*02:07 should also be included in the A2 supertype (46). Ito et al (47) and Nonaka et al (48) reported that an HLA-A2-restricted 
CTL line established from the tumor-infiltrating lymphocytes (TIL) of an HLA-A*02:07-positive patient showed significant cytotoxicities for HLA-A*02:01-, HLA-A*02:06- and HLA-A*02:07-positive cancer cells. Therefore, we examined whether the GPC $3_{144-152}$-specific CTL clone 24-4-2, which was established from the PBMCs of an HLA-A*02:07- positive patient with $\mathrm{HCC}$, could recognize HLA A-A*02:01 or HLA-A*02:06. However, this CTL clone failed to recognize HLA-A*02:01 or HLA-A*02:06.

We have reported previously on the detection via immunohistochemical staining of massive infiltration of CD8-positive $\mathrm{T}$ cells into the remaining liver tumor of this patient (27). It was difficult to confirm that these tumor-infiltrating $\mathrm{CD}^{+}$ $\mathrm{T}$ cells have specificity for GPC3. Currently, we are conducting clinical testing of liver biopsies taken before and after GPC3 peptide vaccination of patients with advanced HCC. Our aim is to reveal the GPC3 peptide-specific immune responses induced by the GPC3-derived peptide vaccine in both the peripheral blood and the tumor. We are analyzing the $T C R$ gene sequences of CD8 or GPC3 dextramer positive T cells in both the peripheral blood and tumor. Already in this trial, a remarkable clinical effect has been observed for an HLA-A*02:07-positive patient with HCC who received GPC $3_{144-152}$ peptide vaccination (49).

HLA-A*02:07 is present in the populations of East Asia, South-East Asia (7\%), and northern India (11.5\%) (26,50-52). In southern China, the frequency of the HLA-A*02:07 allele is reported to be even higher than the frequency of the HLA-A* 02:01 allele $(53,54)$. In addition, about $75 \%$ of liver cancer cases occur in South-East Asia, including China, Hong Kong, Taiwan, Korea, India and Japan (55). Taking together these previous reports and our results, it appears that HLA-A*02:07-positive patients with HCC are good candidates for GPC $3_{144-152}$ peptide vaccination. Further studies will be necessary to prove the clinical efficacy of GPC3 peptide vaccination for advanced HCC.

In conclusion, we present substantial evidence that GPC $3_{144-152}$-specific CTLs with different TCR allele sets that are induced in patients with $\mathrm{HCC}$ who show a PR following GPC $3_{144-152}$ peptide vaccination indicate not only high avidity but also natural antigen-specific killing activity against tumor cells.

\section{Acknowledgements}

We thank Dr Ryo Abe and Dr Toshihiro Suzuki for providing the pcDNA3.1 construct that expresses HLA-A*02:07. This study was supported in part by Health and Labor Science Research Grants for Clinical Research and Third Term Comprehensive Control Research for Cancer from the Ministry of Health, Labor and Welfare, Japan and the National Cancer Center Research and Development Fund.

\section{References}

1. Jemal A, Bray F, Center MM, Ferlay J, Ward E and Forman D: Global cancer statistics. CA Cancer J Clin 61: 69-90, 2011.

2. Ferlay J, Shin HR, Bray F, Forman D, Mathers C and Parkin DM: Estimates of worldwide burden of cancer in 2008: GLOBOCAN 2008. Int J Cancer 127: 2893-2917, 2010.

3. Zidan A, Scheuerlein H, Schüle S, Settmacher U and Rauchfuss F: Epidemiological pattern of hepatitis B and hepatitis $\mathrm{C}$ as etiological agents for hepatocellular carcinoma in iran and worldwide. Hepat Mon 12: e6894, 2012.
4. Llovet JM, Ricci S, Mazzaferro V, et al: Sorafenib in advanced hepatocellular carcinoma. N Engl J Med 359: 378-390, 2008.

5. Cheng AL, Kang YK, Chen Z, et al: Efficacy and safety of sorafenib in patients in the Asia-Pacific region with advanced hepatocellular carcinoma: a phase III randomised, double-blind, placebo-controlled trial. Lancet Oncol 10: 25-34, 2009.

6. Kim HY and Park JW: Molecularly targeted therapies for hepatocellular carcinoma: Sorafenib as a stepping stone. Dig Dis 29: 303-309, 2011

7. Morimoto M, Numata K, Kondo M, et al: Higher discontinuation and lower survival rates are likely in elderly Japanese patients with advanced hepatocellular carcinoma receiving sorafenib. Hepatol Res 41: 296-302, 2011.

8. Greten TF, Manns MP and Korangy F: Immunotherapy of hepatocellular carcinoma. J Hepatol 45: 868-878, 2006.

9. Mizukoshi E, Nakamoto Y, Arai K, et al: Comparative analysis of various tumor-associated antigen-specific t-cell responses in patients with hepatocellular carcinoma. Hepatology 53: 1206-1216, 2011.

10. Filmus J, Shi W, Wong ZM and Wong MJ: Identification of a new membrane-bound heparan sulphate proteoglycan. Biochem J 311: 561-565, 1995.

11. Filmus J and Selleck SB: Glypicans: proteoglycans with a surprise. J Clin Invest 108: 497-501, 2001.

12. Nakatsura T, Yoshitake Y, Senju S, et al: Glypican-3, overexpressed specifically in human hepatocellular carcinoma, is a novel tumor marker. Biochem Biophys Res Commun 306: 16-25, 2003

13. Capurro M, Wanless IR, Sherman M, Deboer G, Shi W, Miyoshi E and Filmus J: Glypican-3: a novel serum and histochemical marker for hepatocellular carcinoma. Gastroenterology 125: 89-97, 2003.

14. Nakatsura T and Nishimura Y: Usefulness of the novel oncofetal antigen glypican-3 for diagnosis of hepatocellular carcinoma and melanoma. BioDrugs 19: 71-77, 2005.

15. Shirakawa H, Kuronuma T, Nishimura Y, et al: Glypican-3 is a useful diagnostic marker for a component of hepatocellular carcinoma in human liver cancer. Int J Oncol 34: 649-656, 2009.

16. Shirakawa H, Suzuki H, Shimomura M, et al: Glypican-3 expression is correlated with poor prognosis in hepatocellular carcinoma. Cancer Sci 100: 1403-1407, 2009.

17. Motomura Y, Senju S, Nakatsura T, et al: Embryonic stem cell-derived dendritic cells expressing glypican-3, a recently identified oncofetal antigen, induce protective immunity against highly metastatic mouse melanoma, B16-F10. Cancer Res 66: 2414-2422, 2006.

18. Motomura Y, Ikuta Y, Kuronuma T, et al: HLA-A2 and -A24-restricted glypican-3-derived peptide vaccine induces specific CTLs: preclinical study using mice. Int J Oncol 32: 985-990, 2008.

19. Iwama T, Horie K, Yoshikawa T, et al: Identification of an $\mathrm{H} 2-\mathrm{Kb}$ or $\mathrm{H} 2-\mathrm{Db}$ restricted and glypican-3-derived cytotoxic T-lymphocyte epitope peptide. Int J Oncol 42: 831-838, 2013.

20. Komori H, Nakatsura T, Senju S, et al: Identification of HLA-A2- or HLA-A24-restricted CTL epitopes possibly useful for glypican-3-specific immunotherapy of hepatocellular carcinoma. Clin Cancer Res 12: 2689-2697, 2006.

21. Nakatsura T, Komori H, Kubo T, et al: Mouse homologue of a novel human oncofetal antigen, glypican-3, evokes T-cell-mediated tumor rejection without autoimmune reactions in mice. Clin Cancer Res 10: 8630-8640, 2004.

22. Imanish T, Akaza T, Kimura A, Tokunaga $\mathrm{K}$ and Gojobori $\mathrm{T}$ : Allele and haplotype frequencies for HLA and complement loci in various ethnic groups. In: HLA 1991. Vol 1. Tsuji K, Aizawa M and Sasazuki T (eds.) Oxford University Press, Oxford, pp1065-1220, 1992.

23. Sidney J, Grey HM, Kubo RT and Sette A: Practical, biochemical and evolutionary implications of the discovery of HLA class I supermotifs. Immunol Today 17: 261-266, 1996.

24. Yasuda N, Tsuji K, Aizawa M, et al: HLA antigens in Japanese populations. Am J Hum Genet 28: 390-399, 1976.

25. Ellis JM, Henson V, Slack R, Ng J, Hartzman RJ and Katovich Hurley C: Frequencies of HLA-A2 alleles in five U.S. population groups. Predominance of $A^{*} 02011$ and identification of HLA-A*0231. Hum Immunol 61: 334-340, 2000.

26. Mehra NK, Jaini R, Rajalingam R, Balamurugan A and Kaur G: Molecular diversity of HLA-A*02 in Asian Indians: predominance of $A^{*} 0211$. Tissue Antigens 57: 502-507, 2001. 
27. Sawada Y, Yoshikawa T, Nobuoka D, et al: Phase I trial of a glypican-3-derived peptide vaccine for advanced hepatocellular carcinoma: immunologic evidence and potential for improving overall survival. Clin Cancer Res 18: 3686-3696, 2012.

28. Yoshikawa T, Nakatsugawa M, Suzuki S, et al: HLA-A2-restricted glypican-3 peptide-specific CTL clones induced by peptide vaccine show high avidity and antigen-specific killing activity against tumor cells. Cancer Sci 102: 918-925, 2011.

29. Nobuoka D, Yoshikawa T, Sawada Y, Fujiwara T and Nakatsura T: Peptide vaccines for hepatocellular carcinoma. Hum Vaccin Immunother 9: 210-212, 2013.

30. Sawada Y, Sakai M, Yoshikawa T, Ofuji K and Nakatsura T: A glypican-3-derived peptide vaccine against hepatocellular carcinoma. Oncoimmunology 1: 1448-1450, 2012.

31. Purbhoo MA,Li Y, Sutton DH, et al: The HLA A*0201-restricted hTERT(540-548) peptide is not detected on tumor cells by a CTL clone or a high-affinity T-cell receptor. Mol Cancer Ther 6: 2081-2091, 2007.

32. Nakatsugawa M, Horie K, Yoshikawa T, et al: Identification of an HLA-A*0201-restricted cytotoxic T lymphocyte epitope from the lung carcinoma antigen, Lengsin. Int J Oncol 39: 1041-1049, 2011

33. Guo Y, Zhu Y and Sun S: Identification and functional studies of HLA-A0201 restricted CTL epitopes in the X protein of hepatitis B virus. Acta Virol 55: 107-115, 2011

34. McKee MD, Roszkowski JJ and Nishimura MI: T cell avidity and tumor recognition: implications and therapeutic strategies. J Transl Med 3: 35, 2005

35. Harada Y and Kawase I: Single cell-based T cell receptor gene analysis reveals existence of expanded WT1 (Wilms' tumor gene) product-specific $\mathrm{T}$ cell clones in leukemia patients but not healthy volunteers. Med J Osaka Univ 50: 1-12, 2007.

36. Tanaka-Harada Y, Kawakami M, Oka Y, et al: Biased usage of BV gene families of T-cell receptors of WT1 (Wilms' tumor gene)-specific $\mathrm{CD}^{+} \mathrm{T}$ cells in patients with myeloid malignancies. Cancer Sci 101: 594-600, 2010.

37. Morimoto S, Oka Y, Tsuboi A, et al: Biased usage of T cell receptor $\beta$-chain variable region genes of Wilms' tumor gene (WT1)-specific $\mathrm{CD}^{+} \mathrm{T}$ cells in patients with solid tumors and healthy donors. Cancer Sci 103: 408-414, 2012.

38. Valmori D, Dutoil V, Lienard D, et al: Tetramer-guided analysis of TCR beta-chain usage reveals a large repertoire of melan-A-specific $\mathrm{CD}^{+} \mathrm{T}$ cells in melanoma patients. J Immunol 165: 533-538, 2000.

39. Mandruzzato S, Rossi E, Bernardi F, et al: Large and dissimilar repertoire of Melan-A/MART-1-specific CTL in metastatic lesions and blood of a melanoma patient. J Immunol 169: 4017-4024, 2002.

40. Zhou J, Dudley ME, Rosenberg SA and Robbins PF: Selective growth, in vitro and in vivo, of individual $\mathrm{T}$ cell clones from tumor-infiltrating lymphocytes obtained from patients with melanoma. J Immunol 173: 7622-7629, 2004.

41. Akiyama Y, Maruyama K, Tai S, et al: Characterization of a MAGE-1-derived HLA-A24 epitope-specific CTL line from a Japanese metastatic melanoma patient. Anticancer Res 29 : 647-655, 2009
42. Saper MA, Bjorkman PJ and Wiley DC: Refined structure of the human histocompatibility antigen HLA-A2 at 2.6 A resolution. J Mol Biol 219: 277-319, 1991.

43. Madden DR, Garboczi DN and Wiley DC: The antigenic identity of peptide-MHC complexes: a comparison of the conformations of five viral peptides presented by HLA-A2. Cell 75: 693-708, 1993.

44. Sidney J, del Guercio MF, Southwood S, Hermanson G, Maewal A, Appella E and Sette A: The HLA-A0207 peptide binding repertoire is limited to a subset of the A0201 repertoire. Hum Immunol 58: 12-20, 1997.

45. Rivoltini L, Loftus DJ, Barracchini K, et al: Binding and presentation of peptides derived from melanoma antigens MART-1 and glycoprotein-100 by HLA-A2 subtypes: implications for peptide-based immunotherapy. J Immunol 156: 3882-3891, 1996.

46. Sette A and Sidney J: HLA supertypes and supermotifs: a functional perspective on HLA polymorphism. Curr Opin Immunol 10: 478-482, 1998.

47. Ito M, Shichijo S, Tsuda N, Ochi M, Harashima N, Saito N and Itoh K: Molecular basis of T cell-mediated recognition of pancreatic cancer cells. Cancer Res 61: 2038-2046, 2001.

48. Nonaka Y, Tsuda N, Shichijo S, et al: Recognition of ADP-ribosylation factor 4-like by HLA-A2-restricted and tumor-reactive cytotoxic T lymphocytes from patients with brain tumors. Tissue Antigens 60: 319-327, 2002.

49. Sawada Y, Yoshikawa T, Fujii S, et al: Remarkable tumor lysis in a hepatocellular carcinoma patient immediately following glypican-3-derived peptide vaccination: an autopsy case. Hum Vaccin Immunother 9: Mar 6, 2013 (Epub ahead of print).

50. Krausa P, Brywka M III, Savage D, et al: Genetic polymorphism within HLA-A*02: significant allelic variation revealed in different populations. Tissue Antigens 45: 223-231, 1995.

51. Chang CX, Tan AT, Or MY, et al: Conditional ligands for Asian HLA variants facilitate the definition of CD8(+) T-cell responses in acute and chronic viral diseases. Eur J Immunol 43: 1109-1120, 2013.

52. Chen KY, Liu J and Ren EC: Structural and functional distinctiveness of HLA-A2 allelic variants. Immunol Res 53: 182-190, 2012.

53. Shieh DC, Lin DT, Yang BS, Kuan HL and Kao KJ: High frequency of HLA-A*0207 subtype in Chinese population. Transfusion 36: 818-821, 1996.

54. Cheng LH, Jin SZ, Gao SQ, Li Z, Zou HY, Wang DM and Wu GG: Difference in HLAA*02 allele distribution between Han populations in south and north China. Di Yi Jun Yi Da Xue Xue Bao 25: 321-324, 2005 (In Chinese).

55. Mohana Devi S, Balachandar V, Arun M, Suresh Kumar S, Balamurali Krishnan B and Sasikala K: Analysis of genetic damage and gene polymorphism in hepatocellular carcinoma (HCC) patients in a South Indian population. Dig Dis Sci 58: 759-767, 2013. 